\title{
Generating Meaning and Visualising Self: Graphic Symbolism and Interactive Online Cartoon
}

\author{
Anne Bamford \\ Andrew Francois
}

Paper Presented at The Eighth International Literacy \& Education Research

Network Conference on Learning, Dimotiko Skolio of Spetses, Spetses, Greece,

4-8 July 2001

\begin{abstract}
From a visual literacy perspective, images engender a diversity of meanings. Cartooning is one form of visual expression that has the ability to present layers of understanding in suggestive forms combining visual and textual literacies. Reading cartoons especially those with editorial or satirical intent, reveal frames of meaning and graphic symbolism encoded through socio-cultural understandings. The use of cartooning as a communicative medium in interactive design allows information to be communicated broadly and rapidly. 'War and Peace' is an example of web-based educational design that uses cartooning as the mode of communication. It exemplifies the way cartooning can efficiently deal with information in a non-linear and framed manner particularly pertinent to interactive communication.
\end{abstract}

\section{BIONOTE}

Anne Bamford has lectured in Art Education and is currently lecturing in interactive media and learning design at the nationally and internationally recognised Institute for Interactive Media and Learning at the University of Technology in Sydney. Anne is chair of the Australian Institute of Art Educators National Research Council and on the editorial committee of Australian Art Education. She is the Director of Visual Arts for the Centre for Research in Education and the Arts. Anne has extensive experience in developing curriculum support material for teachers and interactive media in tertiary teaching and learning. Her current research interests are in visual literacy and adolescents and the use of interactive media in teaching and learning.

Andrew François is currently lead web designer at the Institute for Interactive Media and Learning, University of Technology, Sydney, Australia. A graduate of the Swinbume School of Film and Television he has produced animation, collaborated with artists and has taught subjects in interactive multimedia and web design. He was a participant in the New Talent Pavilion at MILIA'98 in Cannes France and recently a team-member in the award winning Stuff-Art99 online initiative. 


\section{Generating Meaning and Visualising Self: Graphic Symbolism and Interactive Online Cartoon}

Anne Bamford, The University of Technology, Sydney

Andrew Francois, The University of Technology, Sydney

\section{Introduction}

In the interactive environment, speed is everything. Learners are governed by an aesthetic paradigm which operates at the level of immediate satisfaction of desires. They want information obtained via the web to be aesthetically presented, fast and meaningful. To achieve this aim, online cartoons might provide an avenue for addressing the seemingly competing desires of information, speed and aesthetic pleasure. The War and Peace Project utilizes cartoon graphics in an online environment to present news to young children. This paper analyses this site from a visual literacy perspective.

\section{Cartooning as multi-literacy}

Cartooning can be defined as satirical or humorous drawing that often comments or reflects in some way on issues of public interest. Cartoons embody caricature and culturally specific social analysis. There can be multiple meanings or interpretations of cartoons due to the use of visual/textual puns and metaphor.

Egyptians widely used cartoons and caricatures, while the world "cartone" coming from the Italian for paper, was probably first used by Leonardo da Vinci to refer to the quick sketches made prior to the painting of a canvas or fresco (Lester 1995). 
Cartoons may be single or sequential images. Often sequential images are referred to as 'comics', while moving cartoons presented on televisions, video, film or on the computer screens are often refereed to as 'animations'. For the purposes of this paper, the term cartoon is applied to mean all forms of still or moving, single or multi-framed images presented in print or other media, that provide symbolic visual representations. This definition includes caricatures (exaggerated representations), and editorial and political cartoons, where the purpose is to use cartooning conventions to bring into focus social and cultural issues. As Mc Cloud (Mc Cloud 1994: 31) indicates, cartoons are not just a way of drawing, but rather they are a way of seeing. Cartoons provide a space for the consideration of ideas. Cartoons serve to focus attention and provide a medium for forming identities and framing experiences. Cartoons combine symbolic words and pictures in an integrated way to convey narrative, ideas or thoughts.

Cartoons bridge the space between the visual and textual existence of a thing and the realm of ideas.

Cartoons are formed from complex and emotional unions of text. Cartoons can be used to deal with difficult subjects in a way that is non-threatening. Cartoons have a powerful, yet not fully understood, effect. From a purely semiotic point of view, cartoons constitute a highly sophisticated form of communication. Through the combination of text and visual, cartoons are capable of expressing innermost needs and ideas. The process of closure forms a link in cartooned images, allowing the artist to show parts, but the 'reader' to perceive wholes. In this way cartoons act in a balancing act between the visible and the invisible. The reader or viewer is a conscious collaborator in the process of making meaning. Through closure, the viewer reads into the image tacit understandings and notions of sequence, change, time and motion. In an electronic media, the spaces that allow for this closure become fleeting, continuous and virtually imperceptible.

Cartooning, especially responsive and interactive modes of cartooning used on the web, blur traditional notions of literacy. To be able to 'read' an online cartoon, 
a person needs to be textually literate, visually and aesthetically literate and culturally and socially literate within the context in which the cartoon was conceived. In the cartooning form, these literacies operate simultaneously with the viewer's/reader's experiences to create the layers of meaning needed to decode the cartooned image. While traditional print based cartooning combined the visual and the textual, with the socio-cultural to create meaning, online cartooning adds further dimensions as the cartoons are now interactive and responsive, and the cultural context can be the global online community. Hypertext melds text with images and sounds, and expands views of communication.

\section{The educational application of cartoons in an}

\section{online environment}

Interactive multimedia is now widely used in educational settings. Yet little study has been conducted into the construction of visual forms of representation. The inclusion of cartoons and images in a number of educational and commercial sites implies that there exists an expectation that children and adolescents can read and understand the purpose of imagery, can interpret images, are able to determine relationships among images and read layering within images.

With the rise of the use of visual messages in all media, there has been renewed interest in cartoons as a form of social communication. Cartoons form part of contemporary culture, representing a symbolic way of addressing social issues in society. Cartoons tell as much about the culture of the audience for which they are intended as they do about the culture of the artist that produces them. The symbols and stereotypes used in the cartoon must be understood within a particular cultural context. They frequently deal with complex attitudes and issues. They are bound in a political message and the decoding and interpretation of the visual and textual symbols requires a great deal of contextual 
understanding. This paper is particularly focused on the way cartooning can be used to communicate complex social issues to children.

Visual language (Horn 1998) is capable of conveying ideas and concepts in potentially more effective ways than words or images alone. Incorporating the potential of interactive new media and the internet into an entertaining visual interface opens up the possibilities for understanding information in new or even useful ways. Mc Cloud (1994) explores the power of visual language and communication, dissecting the mechanics of comics and cartoons through iconography, metaphor, semiotics and psychology. He argues that comic and sequential art are underrated as art forms and draws our attention to the communicative power of graphic symbolism. Reading his book, (or rather 'viewing' it since it adopts a self-referential comic book format) we are reminded of the potency of using visuals to explain or describe concepts.
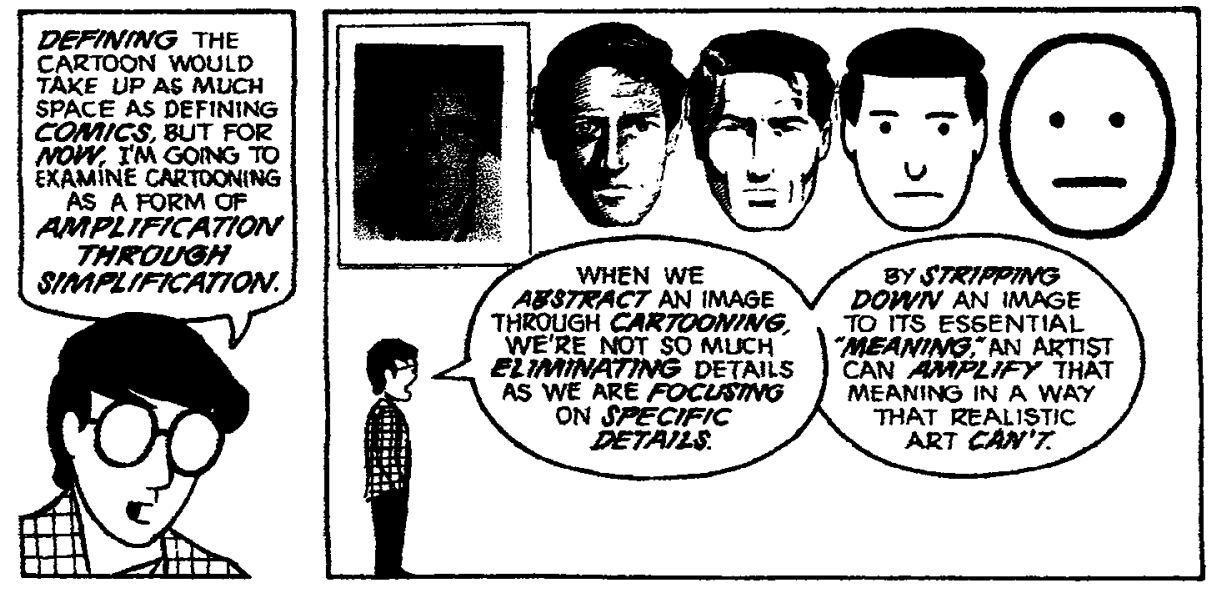

Understanding Comics, Scott Mc Cloud 1993

This ability of the cartoon to 'condense' information clearly makes it an ideal vehicle for conveying ideas on the web. Faced with slow networks and the need for small file sizes the cartoon could be seen as a form of 'intellectual compression', a ready made and space saving alternative to other forms of 
physical graphic compression. An online review

(http://www.nma.gov.au/exhibitions/bthd/index.htm>) of Australian political cartooning states, "Unlike a news article or column, the cartoon has the capacity to almost instantaneously dissect a political issue. [A cartoon] can often have more veracity and insight than hundreds of words of text-based analysis."

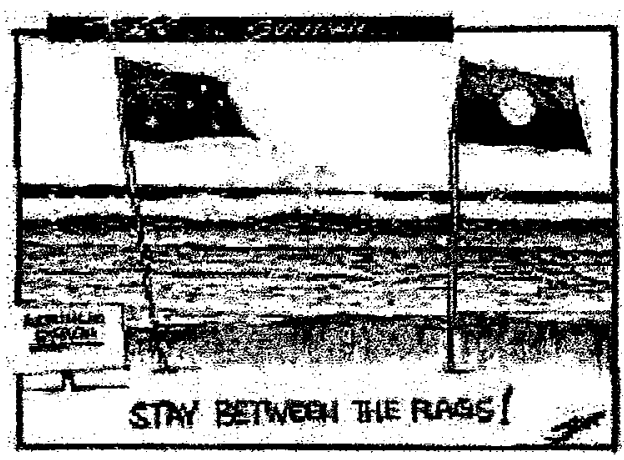

Bringing the House Down: 12 Months of Australian Political Cartoons, National Museum of Australia Exhibition . <http://www.nma.gov.au/exhibitions/bthd/index.htm>

This cartoon examines the political debate in Australia over Aboriginal reconciliation, national identity and the flag itself.

The potential value of cartoons in an educational interactive learning environment is linked to the manner in which they combine text and images to convey complex messages in a short time frame. The use of frames, seriation, text/visual combination and space make cartoons particularly applicable to a learning context based around on screen reading.

The world we see online depends upon how it is framed. Frames are not stagnant entities, but rather shifting structures that serve to organise meaning. Juxtaposition and ambiguity are used to forge connections between seemingly obscure things. In this semantic fusion, the introduction of a context not normally associated with an image serves to establish new target domains, causing the reader to view the original image in a different way. Images as with words can be ambiguous, and it is this ambiguousness that is explored through the development of syntactical frames. These syntactically frames can be gained through proximity of images, chunking, clustering, continuation or sequence of images, establishing a common region, or through the manipulation of prior or 
current associations. Each of these strategies works to encourage the brain to combine elements to create integrated meaning.

Humans naturally have a propensity to group like images together and to make connections between seemingly disparate imagery. For example, when the picture of a car is juxtaposed with the picture of the contented and gorgeous family, the viewer will automatically connect these two ideas, to develop a concept that the car and family exist together as a picture of prestige, safety and personal status. This does not need to be explained, as the presence of the enclosing border in the advertisement, serves to bind these images into single unit of meaning. There is a natural desire to align visuals into a smooth directional continuation, so even without the defining visual frame, people will seek spaces to form this connectiveness. This is very apparent in interactive media, where images are placed in rapid succession to create a 'feel' or emotional connectiveness with the space that has been created on screen. Interestingly, as pop music video clips show very effectively, even when visual stimuli are highly ambiguous, human beings will attempt to make perceptions as "good" as the images allow. People create unity to give the images a sense of closure. This human capacity is exploited in interactive media as it allows the producer of images to play with the intangible to get desired ideas to emerge or be ratified.

Motion and location in space are significant grammars of interactive visual literacy. Increasingly, interactive designers and artists are moving away from the metaphor of the computer screen as a blank canvas. It is no longer regarded as a 2Dimensional space to be filled, but rather interactive artists see locations in space as constructed and manipulable. Objects float randomly in a limitless space created visually by entering multiple coordinates. At the push of a button, flat shapes can be turned into 3-dimensionsal forms that move within a created space. The 'wire frame' allows for the creation of images that can walk, talk and react. Vision is no longer simply a matter of reading a still image in a flat plane. 
Codes of movement and energy are the characteristic of interactive visuals.

Motion and reaction are a significant part of interpreting online images.

Seriation is the manner in which multiple copies of images can be generated saved and manipulating. Unlike traditional ant forms, when working in interactive graphics, it is possible for artists to maintain an infinite number of variations on a piece of work. While art galleries conservers marvel at $\mathrm{x}$-rays of paintings under paintings or preliminary sketches of paintings under oil paint, interactive images exist as multiples. This is significant as seriation of images serves to question notions of originality and 'process versus product' debates in visual communication. With seriation, at any stage the making process can be the product (even retrospectively) and vice versa. Similarly, where one image exists, millions of copies can be made at the touch of a print key.

Text in interactive visuals is kept to the barest minimum. It is used as a design device or where visual reading needs some further support to reduce ambiguities and to increase communicative power. Often only one or two words are used, and it is the size and space of the text that is as important as the meaning of the text itself. Words are associated with visual elements to communicate related information and to clarify the meaning and interpretation of each other. Labeling is frequently used in an interactive environment to assist in the process of forming visual relationships between or within symbolic elements.

Technology related communication is highly complex and dynamic. Information technology has simultaneously become more sophisticated in its application, while becoming more simplified and accessible in its use. Using interactive media everyone becomes both producer and participant in the visual culture.

Adolescents live in a world where visuals are increasingly integrated into the media. The Web, video clips, CD Roms and television are all intensely graphical environments. There has been a proliferation of non-print media, which is interactive and generative. As our society moves forward in the information age,

Leaming for the Future - Proceedings of the Leaming Conference 2001 - 10 
strategies for learning from various forms of texts play an increasingly important role in our conception and definition of what it means to be fully literate. As Smith (1997: 1) indicates "Images are now used primarily as shorthand methods of representing information... increasingly symbolic orientation of communication compels the writing instructor to address the dynamics of visual discourse." The world of the Internet is a fabricated world that has been created to be experienced and interpreted in a particular way. It is a simulated environment that relies heavily upon the power of visual persuasion. Information presented using interactive multimedia is not read in a traditional sense, but rather explored holistically and divergently.

It is argued that online cartoons can effectively address the issues of framing, text as labeling seriation and connectiveness in a way that allows construction of knowledge in an aesthetic and efficient manner. To exemplify this possibility, the design of the War and Peace Project is used as a case study of the enactment of these issues within an online context.

\section{"War \& Peace" - an example of experimental interactive online animated cartoon}

'War \& Peace' provides a forum in which to explore web based cartooned visual representations. Technology related communication using cartoons is highly complex and dynamic. As information technology becomes more sophisticated in its application, greater opportunities to present online cartoons exist. Information presented using interactive multimedia is not read in a traditional sense, but rather explored holistically and divergently. The cartooning medium is ideally suited to this form of exploration as it enables multiple layers of meaning to be presented economically in a single screen shot. The world of the Internet is a fabricated world that has been created to be experienced and interpreted in a particular way. It is a simulated environment that relies heavily upon the power of the visual. Cartoons allow postmodernist concepts such as 
ambiguity, layering, seriation and contextualism to be played out in an interactive educational environment.

Cartoons have always been used as a socio-political device, capable of carrying meaning in layers of depth. In online cartooning visual and print literacies are complementary and interdependent. Information contained on the Web and in interactive media is non-linear. Text is deliberately spaced to break-up connectability and to enable "grab" reading. The space has become more important than the text, as these visual gaps encourage the process of ideation. Knopfer's (1997) study of web sites found that non-educational commercial sites tended to foreground graphics and animations, and that these were most favoured by adolescents. Educational sites have tended to lag behind game and recreational web sites in the use of graphics and have tended to favour traditional linear text and were not favoured by students.

'War \& Peace' is an experimental interactive online animated cartoon that is used on an educational news site to encourage students to become actively engaged in current affairs.

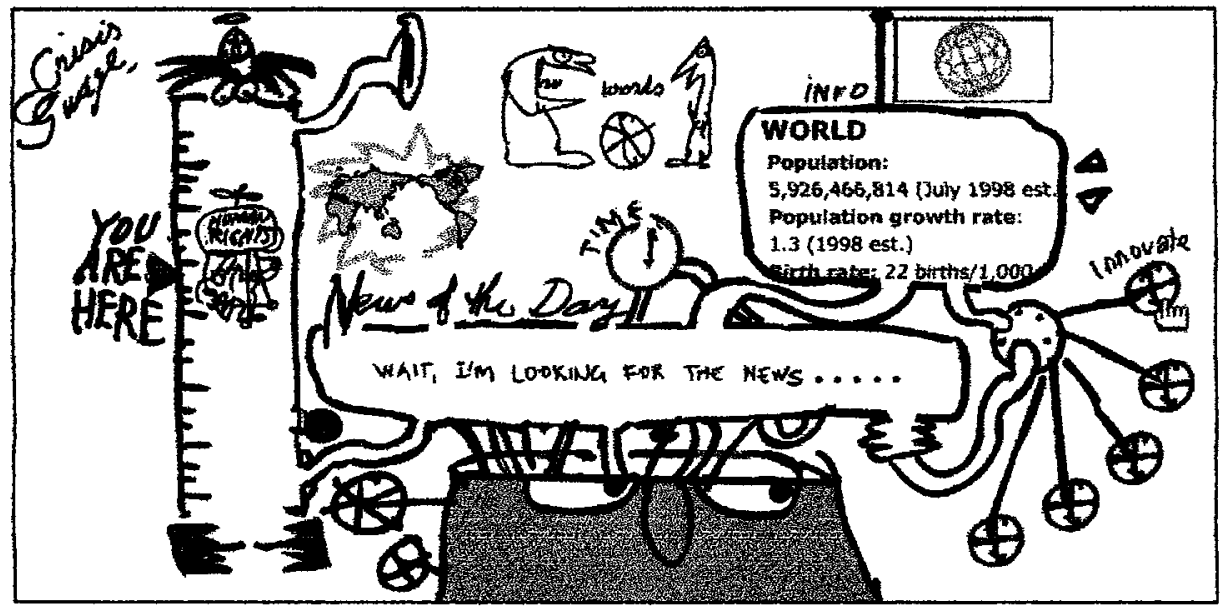

< http:/hwww,abc.net.au/arts/stuff-art/stuff-art99iwar.htm> @1999 
In 'War \& Peace' (see example above), a Bruce Petty cartoon, comes to life in an exploration of humanity's apparent disposition towards violence. It is designed to be viewed solely on the web and is framed both physically and metaphorically to accommodate the affordances of onscreen reading. This cartoon is a lighthearted interactive exploration of the heavy subjects of war, peace and conflict resolution. The intended audience includes children and adolescents. The viewers are confronted by the concept that there is a basic dominant human disposition towards violence and that peace is not a natural condition but instead requires constant monitoring and imaginative, ongoing effort. The aim for the child is to avoid war. Adjusting the diplomatic valves applies pressure to get the 'Crisis Gauge' moving, ideally towards peace. Two generic characters at the top of the contraption respond to the present state of conflict, reflecting the level of the 'Crisis Gauge'. Live online news headlines give the contraption an alarming measure of relevance. Real background data downloaded from the online CIA World Factbook on countries featured in these headlines can also be viewed. 'War \& Peace' proposes that for peace to become a natural human destination it must become, by some major attitude shift, as interesting as war.

\section{Rationale}

Conceived of as a prototype educational 'tool' War and Peace acts as a catalyst in social debate, operating under the premise that humour and satire can be used as instruments for social change. It attempts to facilitate the construction of meaning of events in contemporary culture through the recontextualisation of the world news. 


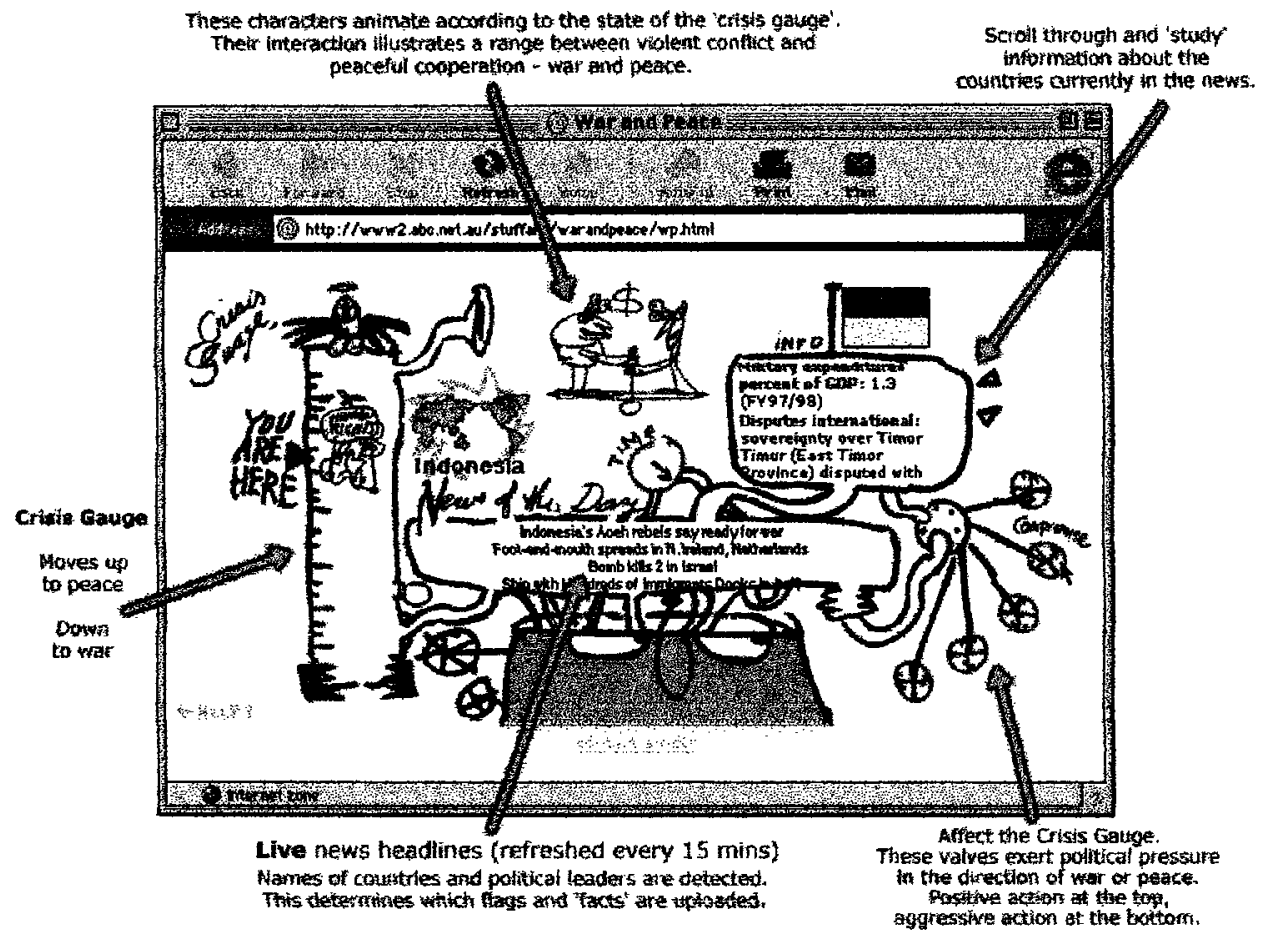

The intention is that interactive cartoons could be more extensively used as an online teaching aid to help make current affairs and critical social analysis both interesting and fun for participating students. News feeds, stock market figures, statistics and data of all kinds could be visualised and examined from a new perspective.

\section{Deconstructing cartoons}

Cartoons, while seemingly packaged in a deceptively simple frame, represent, "a complex exercise in semiotic analysis" (Lester 1995: 244). Cartoons utilise a number of narrative and artistic devices are particularly suited to online construction of knowledge. Cartoons are largely constructed of icons. The abstracted icons are used to resemble reality, rather than represent it. Cartoons use both visual and textual clues to construct metaphors, whereby, "one idea is used to represent a second meaning or idea in order to suggest an analogy or 
likeness" (Horn 1998: 106). The range of visual and textual devices can include such things as voice, humour, setting, irony, mood, impact, source, typography, sequence, voice, caption or situations.

The conventions of cartooning that are particularly pertinent to online communication include aspects such as;

- visual representation of transitions in time and space;

- expressive line or shape to show feelings or emotions;

- visual presentation of physical phenomenon;

- action lines to indicate movement or gestures;

- thoughts and emotions expressed through physical representation of faces; and

- integration of visual and text.

By deconstructing an example of cartooning used in an educational online environment, it is possible to understand the nature of visual literacy as applied in interactive technologies. This study aims to identify and define the visual literacy constructs applied in one example of online cartooning.

The technology of the World Wide Web offers different affordances to the traditional print based cartoon media of newspapers and magazines. In online cartooning, space is less defined and cartoons are adopting non-linear compositions. The visual graphic packages encourage the development of layered cartoons. The cartoons may be more multi-dimensional. There is greater opportunity to integrate sound and movement. Boxes and panels have largely disappeared and the structure of cartoons has become more holistic, as the computer screen itself becomes the 'frame' for the narrative. The disappearance of frames brings the creator and the viewers into a more intimate relationship. The technology also allows moving between a range of way images in cartoons are framed. For example, things in the centre can be moved to the margins, while an image in close focus can be shifted to far away. Similarly, while the paper-based cartoon is generally read from left to write, interactive cartoons 
can be read non-linearly, with a range of active 'hit' points that allow the audience to more directly contribute to the construction of the message and/or image.

The role of text is particularly important in online cartoons. In this environment speed and audience attraction are everything, and so the general movement has been away from reading of sections of text, to text being used as labels. In popular culture, words and pictures work together to communicate ideas. In the example of War and Peace, words have been used in an additive way, such as when they amplify or elaborate on the ideas expressed in the visuals of the cartoon. They have also been used in parallel to the visuals, as is exemplified in cartoon where label in the text runs adjacent to the images, and our only reveled through the action of scrolling the mouse. Increasingly words are used in online cartooning in a montaged fashion, where the words and pictures operate in an integral and interdependent manner to convey ideas. In War and Peace, it is impossible to separate the words and pictures. Together they form the narrative and meaning of the cartoon. The words and pictures work together in War and Peace to explore internal dialogues, expand incongruence, amplify emotional information or express changes in time or place. The power of integrating text and imagery is not new. Asian culture has long identified the link between writing and the visual. Yet this link is less obvious in the ways in which western education deals with literacy. As Lester (1995: 417) cautions:

We are taught to distinguish words from pictures and to not think of them in the same way. We were taught that, although we would gain meaning from each, reading words was valued more than reading pictures. We are taught that pictures play a separate and subservient role to the words. And although we learned how to make pictures with coloured pencils and our water-colour paints, we received much more instruction on how to form, with our large lead pencils, the lines and curves that usually make letters and words. We usually had one class where we made pictures - art. The other classes were devoted to writing or reading stories, whether in a grammar or in a geography class. We were taught to read stories but we're never taught how to read images. 
In the online environment, both words and visuals must be stripped down to their simplest and most quickly accessible symbolic forms. As Mc Cloud (1994: 49) notes, "When pictures are more abstracted from 'reality' they require greater levels of perception, more like words. When words are bolder more direct, they require lower levels of perception and are received faster, more like pictures." The increasing use of the visual in education, and online cartooning specifically, indicate the importance of educating children to read the visual. Online cartoons provide a device that is capable of amplifying ideas and embodying complex concepts. Through simplified, subtractive symbolism, online cartoons are able to focus children's attention on specific details. As Mc Cloud (1994: 30) notes, "By stripping down an image to its essential "meaning" an artist can amplify that meaning in a way that realistic art can't". The simplified, schematic drawings, as used in the 'War \& Peace' online cartoon, reveal information to the viewer. The viewer readily recognises the properties of the depicted and often distorted caricatures. With seeming ease, highly sophisticated readings can be made, that the text alone would not enable.

\section{Cartoons as social records and catalysts}

Cartooning, as an art form, is socially and culturally defined by the context in which it was created. Yet its very specificity enhances its universality with cartoons being able to speak of our greatest fears and love and be capable of marking our identity as humans and instill in us a binding sense of community and reciprocity. Cartoons allow for the exploration of the non-verbal parts of our minds and develop ways of learning within the context of multi-layered symbols and rich meanings.

Cartoons frequently articulate life concerns. They acknowledge those things that we most care about. Through the use of visual devices and visual and textual satire cartoons frequently comment upon the deeper and higher parts of human nature. As is evident in War and Peace, significant cartoons transcend the 
individual, time and circumstance to express feeling and transmit understanding, across cultural and historical boundaries, speaking to all people. Aarons (1991) described art as 'soul or thought prints in the sands of time'. Similarly, cartoons in an interactive environment may assist in building knowledge in the global village by having the ability to speak to people simultaneously at a very personal and yet universal manner. Through interactive cartoons it may be possible to understand complexities and relationships otherwise beyond human grasp. Cartoons can convey both shared universal and specific culturally bound meanings.

\section{Conclusion}

'War \& Peace' is an experiment in editorial cartooning for the online news and current affairs of the future. The traditional daily editorial cartoon relies in part on the authenticity, immediacy and currency of its subject matter for much of its strength. By drawing upon the Internet as a resource for 'up to date' news and information we are continuing to investigate how an interactive cartoon might be used in an educational environment and to what benefit.

Moving off the printed page into the web browser has allowed the elements of online media (image, text, sound, animation and interactivity) to combine with the traditional cartoon form. 'War \& Peace' as an experiment hopes to lead the way toward a new kind of cartoon where meaning is generated at the point where the student, live data and new media converge.

Cartoons such as War and Peace, open new possibilities for forms of literacy and communication to develop that more appropriately address the affordances of an onscreen environment. The traditional nature of cartoons, including the strong visual text relationship, the framing of narrative, the use of text as labels and the seriation and layering of ideas are powerful tools in online communication. The educational use of online cartoons are only beginning to be explored. In addition 
to the design attributes of cartooning that make them a particularly suitable medium of interactive environments, of greater significance may be the universal appeal of cartoons and the way they reach out aesthetically to their audience. As in War and Peace, the cartoon may provide an avenue for dealing with complex issues in a way that is appealing and accessible. In presenting our culture both visually and textually, it may in fact also communicate notions of self in a way that is frequently felt to be lacking in an online environment. 


\section{References}

Aarons, A. (1991). "A sociology of art...why does art look (or sound) like it does?" Artlink 11(3): 36-39.

Horn, R., E. (1998). Visual language: Global communication for the 21st Century. Bainbridge Island, Washington, MacroVU, Inc.

Knopfer, N. and e. al (1997). Visual aesthetics and functionality of web pages: Where is design? National Convention of the association for educational communications and technology, Albuquerque, ED 409846.

Lester, P. M. (1995). Visual communications: Images with messages. Belmont, California, Wadsworth Publishing Company.

Mc Cloud, S. (1994). Understanding comics: The invisible art. New York, Harper Collins.

< http://www.acn.net.au:80/articles/1998/12/cartoon.htm > 


\section{Technical details}

'War \& Peace' interactive online animated cartoon

Size:

433k Shockwave .dcr file plus $805 \mathrm{k}$ of linked external data (world flags and statistics).

Format:

Shockwave 7 (Browser plug-in required)

Software used:

Macromedia Director7, Macromedia Flash3, Adobe Photoshop5, Adobe ImageReady1, Macromedia Soundedit 16.

Hardware used: Macintosh 8600/200/112 meg, Macintosh G3/233/128meg,

Wacom graphics tablet, Dual $266 \mathrm{mhz}$ Pentium-NT workstation. 
This paper is published at http:/LeamingConference.Publisher-Site.com/ a series imprint of the UniversityPress.com

First published in Australia in 2002 by Common Ground Publishing Pty Ltd at http:/LeamingConference.Publisher-Site.com/

Selection and editorial matter copyright 0 Bill Cope and Mary Kalantzis 2002 Individual chapters copyright $\odot$ individual contributors 2002

All rights reserved. Apart from fair dealing for the purposes of study, research, criticism or review as permitted under the Copyright Act, no part of this book may be reproduced by any process without written permission from the publisher.

ISBN 1863352783 (print)

ISBN 1863352791 (PDF) 
Generating Meaning and Visualising Self: Graphic Symbolism and Interactive Online Cartoon

Anne Bamford Andrew Francois
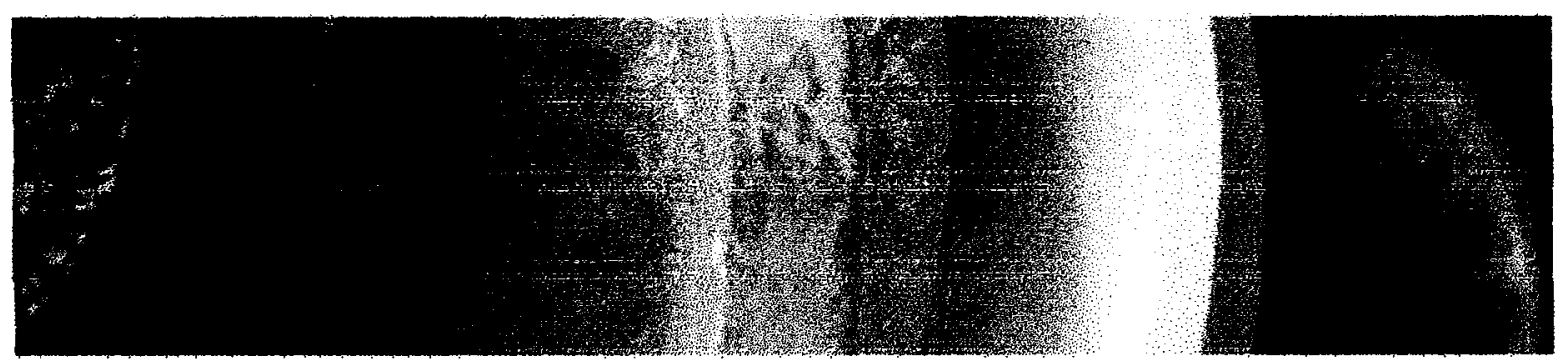

Edited by Bill Cape and Mary Katantzis

\section{LEARNING FOR THE FUTURE}

Proceedings of the Leaming Conference 2001 


\title{
Generating Meaning and Visualising Self: Graphic Symbolism and Interactive Online Cartoon
}

Anne Bamford Andrew Francois

\begin{abstract}
Paper Presented at The Eighth International Literacy \& Education Research Network Conference on Learning, Dimotiko Skolio of Spetses, Spetses, Greece, 4-8 July 2001

Offprint from:

Leaming for the Future Proceedings of the Learning Conference 2001

Edited by Bill Cope and Mary Kalantzis
\end{abstract}
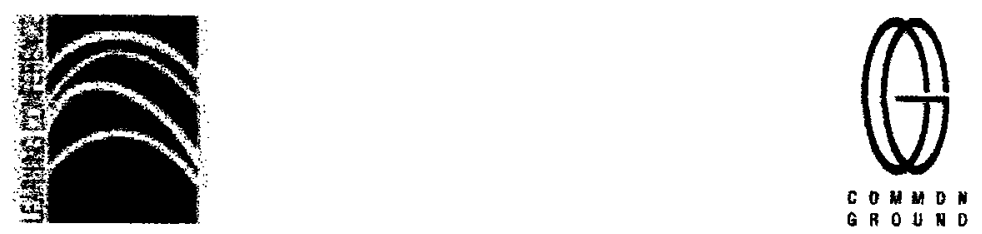

www.LeamingConference.com

www.thelearner.com 